Journal of Animal and Veterinary Advances 11 (14): 2533-2539, 2012

ISSN: $1680-5593$

(C) Medwell Journals, 2012

\title{
Anatomic Peculiarities of the Cerebral Arterial System and Blood Supply in the Yak (Bos grunniens)
}

\author{
${ }^{1}$ X.R. Wang, ${ }^{2}$ Y. Liu, ${ }^{3}$ T.F. Guo and ${ }^{1}$ J.P. Wu \\ ${ }^{1}$ College of Animal Science and Technology, ${ }^{2}$ College of Animal Veterinary, \\ Gansu Agricultural University, 730070 Lanzhou, China \\ ${ }^{3}$ Lanzhou Institute of Animal and Veterinary Pharmaceutical Science, CAAS, 730050 Lanzhou, China
}

\begin{abstract}
Anatomic characteristics of the cardiovascular system can reflect different supplying blood ability to tissues and organs. The Cerebral Arterial System mainly contributes to supplying blood to the brain tissue. In present study, researchers examined the morphological features and internal diameters of arteries and analyzed supplying blood ability of the Cerebral Arterial System of the yak and Chinese cattle with the Corrosion Casting technique and the methods of comparative anatomy. Results showed that general arrangements and calibers of most arteries of the Cerebral Arterial System between two species were similar but the anterior-posterior diameter of the cerebral arterial circle was shorter and interval distance of the cerebral carotid artery was closer in the yak. The yak has more developed internal arterial vessels and epidural retia mirabile and the blood volume of the Cerebral Arterial System in the yak showed anatomical superiority. Researchers deduced that the anatomic peculiarities of the cerebral arterial circle and relatively developed internal arterial vessels in the yak were beneficial to supplying more blood to the brain. The yak's developed epidural retia mirabile was advantageous of the blood preservation, exchange and complement and it might play a vital role in buffering and regulating cerebral blood flow so as to meet demand of the yak's brain for blood supply in the plateau areas.
\end{abstract}

$\underline{\text { Key words: Yak, anatomic peculiarities, Cerebral Arterial System, brain, China }}$

\section{INTRODUCTION}

The Cardiovascular System carries the blood to all of organs and tissues which ensure to meet their demand for oxygen and nutrient. The brain is the center of the nervous system in all vertebrate animals and its blood supply mainly via the Cerebral Arterial System (CAS). Previous studies showed not only the anatomic characteristics of arteries accounted for different supplying blood ability but environmental factors also contributed to different hemodynamics. Some studies found that the diameter of the internal carotid artery in Tibetans and Han Chinese was similar but the cerebral blood flow response to exercise was significantly different (Huang et al., 1992). Himalayan populations have substantially higher brain blood flow than Andean populations (Jansen and Basnyat, 2011). The yak (Bos grunniens) has lived in low oxygen and high altitude areas such as Qinghai-Tibet plateau for a long time. At present, more and more studies have focused on the plateau adaptability of the yak and mainly concentrated on blood physiology and anatomical features of the heart, lung vessels (Lalthantluanga et al., 1985; Cui et al., 2008; Du, 2008; Wang et al., 2012). Still there were many studies in cattle reported morphological characteristics of the cerebral arteries (Baldwin and Bell, 1963; Baldwin, 1964; Ocal and Aslan, 1994; Kurtul et al., 2003) and analyzed differences of anatomical features of the CAS between cattle and other domestic animal (Shwini et al., 2008). Besides, some studies described structural peculiarity of the Epidural Retia Mirabile (ERM) and the pathway of blood supply in ox and calf (Steven, 1964; Uehara et al., 1978). But reports about the yak's anatomical peculiarities of the CAS associating with the cerebral blood supply were very seldom.

Study of anatomic differences on the CAS between the plateau yak and low-altitude Chinese cattle (Bos taurus) will help to reveal yak's tolerance mechanisms to hypoxemic and hypobaric environments. In this research, we examined anatomical peculiarities of the CAS and analyzed the blood supply features to the brain between the two species, aiming at providing a morphological basis for further research in the yak and exploring the yak's plateau adaptability of blood supply to the brain.

Corresponding Author: Y. Liu, College of Animal Veterinary, Gansu Agricultural University, 730070 Lanzhou, China 


\section{MATERIALS AND METHODS}

Twelve heads from healthy and adult yaks living in Qinghai (altitude is $3,800 \mathrm{~m}$ ) were collected immediately after slaughter and ten heads from Chinese cattle were collected in Gansu (altitude is 1,800 m). Animals were killed by exsanguination for food and their sex and weight were not recorded. Specimens were demanded fresh, intact and pollution-free. Perfusion Methods were carried out as described by $\mathrm{He}$ (2007) and the ABS solution (20\% ABS in butanone) was injected into the cerebral arterial vessels for $200-300 \mathrm{~mL}$ via the common carotid artery until the vertebral artery was seen very full through the foramen magnum then specimens were kept for 2-3 days. After casting solution was totally hardened, the skin and muscle around the skulls were peeled off manually and taken off redundant organs and tissues outside the cranial cavity so as to ensure the brain tissue and adjacent vessels intact.

At last, specimens were soaked in the concentrated hydrochloric acid for 7-10 days. After having finished corrosion, remnants of brain tissue were carefully flushed with pipe water until vascular casts were emerged totally. Specimens' figures were obtained by digital camera and their measurements such as diameters and sizes were determined by vernier caliper. The morphological parameters of the CAS measured in present study were as follows:

- Interval distance of two Cerebral Carotid Arteries (CCA) within cranial cavity

- The morphological features of the Cerebral Arterial Circle (CAC) including the anterior-posterior and left-right diameters of the anterior circle $(\mathrm{AC}$, means the partial circle anterior to the $\mathrm{CCA}$ ) and the posterior circle ( $\mathrm{PC}$, means the partial circle posterior to the $\mathrm{CCA}$ )

- Morphological sizes of the ERM included the top width, middle width and bottom width

In addition, the internal diameters of main arteries were measured at the origin of vessels in CAS and their significance of differences were analyzed by SPSS Software.

\section{RESULTS}

General features of the CAS: The CAS of the yak and cattle were divided into three parts: the Intracranial Arterial Vessels (IAV, Fig. 1A), the REM (Fig. 1B) and the Extracranial Arterial Vessels (EAV, Fig. 1C). The IAV lied inside the cranial cavity which was composed of the CCA,
$\mathrm{CAC}$ and cerebral arterial branches originating from the $\mathrm{CAC}$ and was the direct pathway of supplying blood to the brain. The ERM lay on the floor of the cranium and outside dura mater was an anastomotic network partially lying in the cavernous sinus.

The ERM was regarded as a blood collecting centre at the bottom of the brain and there were plenty of complex anastomotic branches in it. Its blood supply mainly came from the maxillary, occipital and vertebral arteries. After arriving at the meshwork, arterial blood was collected and mixed together and supplied to the CAC mainly by the CCA penetrating the dura mater to enter the brain. The EAV lay outside the cranial cavity, it was the main channel of carrying blood to the brain from heart. Casts showed that the EAV was consisted of the maxillary, external ophthalmic, external carotid, internal carotid, vertebral, occipital and common carotid arteries. The blood was supplied to different section of the ERM via the EAV, so it was regarded as the indirect pathway of supplying blood to the brain.

The main pathway of the arterial supply to the brain in the yak and cattle was: heart $\rightarrow$ common carotid artery $\rightarrow$ external carotid artery $\rightarrow$ maxillary artery $\rightarrow \mathrm{ERM} \rightarrow \mathrm{CCA} \rightarrow$ $\mathrm{CAC} \rightarrow \mathrm{IAV} \rightarrow$ brain tissue. In addition, the occipital, vertebral, internal carotid and condylar arteries were also collateral supplying blood vessels to the brain (Fig. 1A-D).

Morphological comparison of the CAC: The shape of the $\mathrm{CAC}$ in the yak and cattle looked like an inverted bottle gourd and their basic formation were similar. The CAC was formed by five arteries: the anterior communicating, anterior cerebral, posterior communicating, basilar arteries and CCA. The main arteries locating at the $\mathrm{AC}$ and originating from it were the anterior communicating, anterior cerebral, middle cerebral and anterior choroidal arteries. The main arteries locating at the PC and originating from it were the posterior cerebral, posterior communicating, posterior choroidal, anterior cerebellar and basilar arteries. The main arteries originating from the basilar artery were the posterior cerebellar artery and other small arteries (Fig. 1A and B).

Morphological features of the $\mathrm{AC}$ and $\mathrm{PC}$ between the yak and cattle exhibited a little difference. Though the left-right diameter of the $\mathrm{AC}$ was similar in two species, the left-right diameter of the PC in the yak was slightly wider than that of cattle. Anterior-posterior diameter of the CAC in the yak was significantly shorter than that of cattle (Table 1). To the morphological features of the CAC in the yak, researchers found that the $\mathrm{AC}$ was flatter and the PC looked like radish shaped meanwhile its blood vessels' course was more flexuous than that in cattle (Fig. 1A). The PC of cattle showed the inverted triangle 

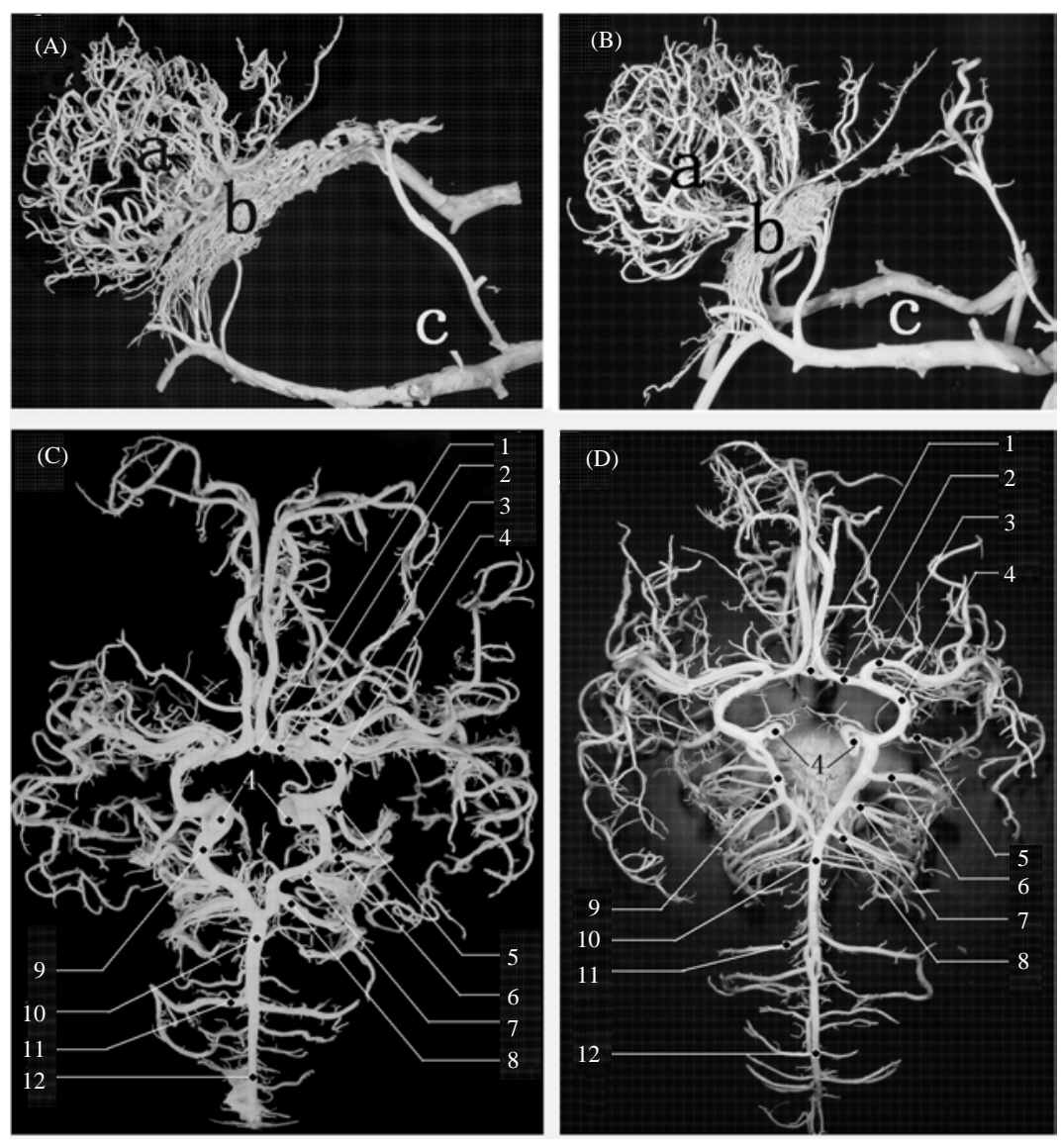

Fig. 1: Corrosion casts from the CAS of the yak and cattle: A) CAS of the yak; B) CAS of cattle; C) IAV of the yak; D) IAV of cattle; a) IAV; b) ERM; c) EAV (CAS = Cerebral Arterial System; IAV = Intracranial Arterial Vessels; $\mathrm{ERM}=$ Epidural Retia Mirabile and EAV = Extracranial Arterial Vessels $) .1=$ Anterior communication artery; 2 = Anterior cerebral artery; 3 = Middle cerebral artery; 4 = Cerebral carotid artery; 5 =Anterior choroidal artery; $6=$ Posterior cerebral artery; $7=$ Posterior choroidal artery; $8=$ Anterior cerebellar artery; $9=$ Posterior communication artery; $10=$ Basilar artery; $11=$ Posterior cerebellar artery and $12=$ Ventral spinal artery

Table 1: Morphological measurements ( $\mathrm{mm}$ ) of the CAC and ERM in the yak and cattle

\begin{tabular}{lrrr}
\hline Measurements parameters & Yak & Cattle & Yak:Cattle \\
\hline CAC & & & \\
Left-right diameter of AC & 26.98 & 26.73 & $1.01: 1$ \\
Left-right diameter of PC & 20.48 & 18.98 & $1.08: 1$ \\
Interval distance of CCA & 9.32 & 12.07 & $0.77: 1$ \\
Anterior-posterior diameter of AC & 9.10 & 14.99 & $0.61: 1$ \\
Anterior-posterior diameter of PC & 12.33 & 15.34 & $0.80: 1$ \\
Anterior-posterior diameter of CAC & 21.43 & 30.33 & $0.71: 1$ \\
ERM & & & \\
Anastomotic branches of maxillary artery (n) & 6.73 & 5.22 & $1.29: 1$ \\
Cross area of anastomotic branches (mm $\left.{ }^{2}\right)$ & 9.66 & 8.85 & $1.09: 1$ \\
Top width of anterior ERM & 54.57 & 50.53 & $1.08: 1$ \\
Middle width of anterior ERM & 33.98 & 30.23 & $1.12: 1$ \\
Bottom width of anterior ERM & 28.66 & 36.09 & $0.79: 1$ \\
Communicating rami of posterior ERM (n) & 5.43 & 3.21 & $1.69: 1$ \\
\hline CAC
\end{tabular}

$\mathrm{CAC}=$ Cerebral Arterial Circle; ERM $=$ Epidural Retia Mirabile; $\mathrm{AC}=$ Anterior Circle; $\mathrm{PC}=$ Posterior Circle and $\mathrm{CCA}=$ Cerebral Carotid Artery

shaped, meanwhile its blood vessels' course was almost straight (Fig. 1B). In addition, the interval distance of the CCA in the yak was closer than that in cattle (Table 1).
Morphological comparison of the ERM: The general architectures of the ERM were similar in two species, mainly composed of the anterior ERM, posterior ERM, $\mathrm{V}$-shaped extension and basi-occipital arterial plexus (Fig. 2a, b).

The anterior ERM was the most developed and main portion among them. The anterior ERM jointed with the anastomotic branches from the maxillary and external ophthalmic arteries while the posterior ERM joined with the basi-occipital arterial plexus formed by the occipital and vertebral arteries and there was no obvious range between the anterior and posterior ERM. The anastomotic network of the anterior ERM in the yak was thick while the posterior ERM was of single layer. The anterior ERM completely surrounded the pituitary gland and the anastomotic branches prior to it were called the anterior joint section while the posterior to it were called the posterior joint section. 

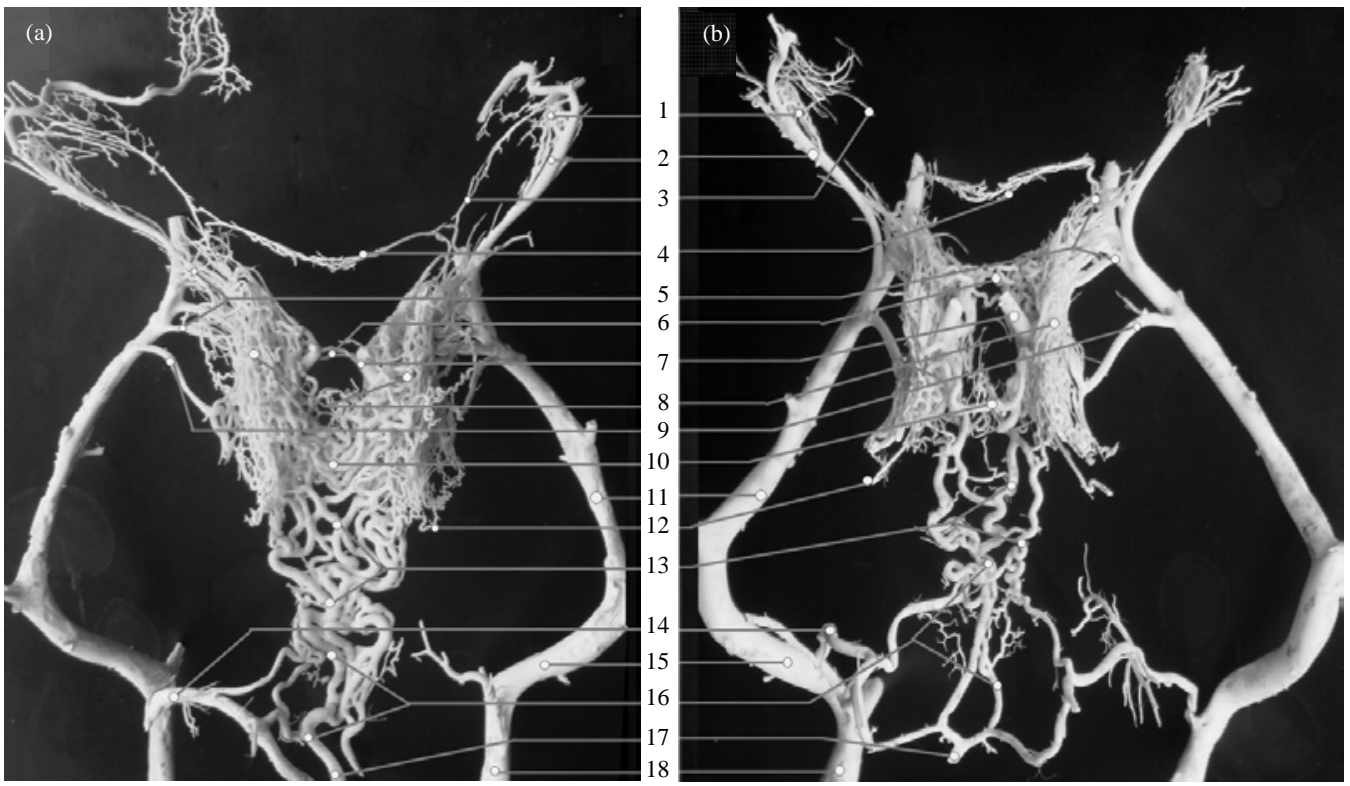

Fig. 2: Corrosion casts from the ERM in the yak and cattle. a) Yak; b) Cattle, $1=$ Ophthalmic rete; $2=$ External ophthalmic artery; $3=$ Internal ophthalmic artery; $4=\mathrm{V}$-shaped extension; $5=$ Anterior anastomosis; $6=$ Anterior joint section; $7=$ Cerebral carotid artery; $8=$ Anterior RME; $9=$ Posterior anastomosis; $10=$ Posterior joint section; $11=$ Maxillary artery; $12=$ Internal carotid artery; $13=$ Posterior RME and communicating rami; $14=$ Occipital artery; $15=$ External carotid artery; $16=$ Basi-occipital arterial plexus; $17=$ Vertebral artery and $18=$ Common carotid artery

The anterior ERM was consisted of two lobes connected by the anterior and posterior joint sections. The V-shaped extension lay at the bottom of the optic chiasma and its left and right sides jointed with the maxillary and internal ophthalmic arteries. The basioccipital arterial plexus located at the distal end of the posterior ERM.

The top and middle width of the anterior ERM in the yak were wider and the bottom width was narrower than that of cattle, so the anterior ERM was close to letter V (Table 1 and Fig. 2a). The bottom width of the anterior ERM in cattle was wider than that of yak and its shape was approximate to letter $\mathrm{H}$ (Table 1 and Fig. 2b). The number of the anterior anastomotic branches from the maxillary artery in the yak was 6.73 on average, significantly more than that in cattle and the gross cross area of the anterior anastomotic branches in the yak were larger than that of cattle (1.09:1). The posterior joint section of the anterior ERM was more developed and the communicating rami of the posterior ERM were abundant (5.43) and arranged densely in the yak while the communicating rami were scarce (3.21) and arranged sparsely in cattle (Table 2).

Arterial diameters and supplying blood features: The average diameter of the $\mathrm{CAC}$ in the yak was a little thinner
Table 2: Internal diameters of the origin of main arteries of the CAS in the yak and cattle

\begin{tabular}{lcc}
\hline Main arteries of CAS & Yak & Cattle \\
\hline IAV & & \\
Anterior cerebral artery & $1.68 \pm 0.25$ & $1.67 \pm 0.31$ \\
Middle cerebral artery & $1.54 \pm 0.18$ & $1.79 \pm 0.33^{*}$ \\
Cerebral carotid artery & $2.32 \pm 0.41$ & $2.39 \pm 0.37$ \\
Posterior cerebral artery & $1.15 \pm 0.21$ & $1.29 \pm 0.27$ \\
Posterior communication artery & $1.66 \pm 0.33$ & $1.98 \pm 0.41^{*}$ \\
Anterior cerebellar artery & $1.07 \pm 0.25$ & $1.21 \pm 0.23$ \\
Basilar artery & $1.76 \pm 0.30$ & $1.85 \pm 0.30$ \\
Posterior cerebellar artery & $0.95 \pm 0.28$ & $1.05 \pm 0.18$ \\
Average diameter of circle & $1.66 \pm 0.23$ & $1.88 \pm 0.31$ \\
ERM & & \\
Anterior anastomosis of maxillary artery & $1.62 \pm 0.33$ & $1.86 \pm 0.43$ \\
Posterior anastomosis of maxillary artery & $1.49 \pm 0.34$ & $1.96 \pm 0.37^{* *}$ \\
Communicating rami of posterior ERM & $1.69 \pm 0.28$ & $1.75 \pm 0.38$ \\
EAV & & \\
External ophthalmic artery & $1.84 \pm 0.28$ & $1.88 \pm 0.35$ \\
Maxillary artery & $6.00 \pm 0.81$ & $5.34 \pm 0.82$ \\
External carotid artery & $7.33 \pm 0.99$ & $6.84 \pm 1.29$ \\
Occipital artery & $1.82 \pm 0.20$ & $2.12 \pm 0.21$ \\
Common carotid artery & $8.21 \pm 1.28$ & $8.97 \pm 2.49$ \\
\hline
\end{tabular}

${ }^{*} \mathrm{p}<0.05 ; * * \mathrm{p}<0.01$; Values are given as Mean \pm SD $(\mathrm{mm})$

than that in cattle and the diameters of constituting vessels of the $\mathrm{CAC}$ and most arteries originating from it in the yak were slightly thinner than those in cattle with no significant differences observed $(p>0.05)$. Researchers found that only the diameters of the middle cerebral and posterior communicating arteries were significantly thinner than that in cattle $(\mathrm{p}<0.05)$. Diameter of the 
posterior anastomotic branch of the anterior ERM in the yak was very significant thinner than that in cattle $(p<0.01)$ and diameters of communicating rami of the posterior ERM were similar between the two species ( $>0.05$ ). The diameters of main vessels of the EAV in the yak were slightly thinner than that in cattle with no significant differences observed ( $p>0.05$, Table 2 ).

Due to diameters measurements of the common carotid, external carotid, maxillary, external ophthalmic arteries and the CCA, we found that decreasing gradient of the next caliber was $10.73,31.86,77.62$ and $71.72 \%$ in the yak, respectively which were milder than that in cattle. In addition, by the method of calculating casts density, we found that blood volume of the IAV was smaller but those of the ERM in the yak was distinctly larger than those in cattle as a result the relative gross blood volume ratio of IAV and ERM between the yak and cattle was $0.966: 1$.

\section{DISCUSSION}

Anatomical peculiarities of the CAC: Results of this research visualized at the yak's cerebral arterial vasculature by corrosion casting and clearly showed the yak's general arrangement of the CAS. By comparison, researchers considered that the morphological features, vessel formation and originating places of main arteries in the CAC of the yak were similar to that seen in cattle, however the anatomical morphologies of the $\mathrm{CAC}$ in the yak showed distinct peculiarities. A study found that different incidences of types of the circle posterior part are obvious as from continent to continent, as from the city to city in same country and another study considered that the relative contribution of each of the proximal arteries correlated significantly with variations in the circle of Willis (Tanaka et al., 2006). These results confirmed the peculiarities of the CAC or distribution of the cerebral blood flow was different among mammalian living in different environments. In present study, it indicated that the more curving vessels' course of the CAC in the yak was advantageous of making blood flow slow down and more blood to be distributed to the brain.

In addition, the study illustrated that diameters of most arteries from the $\mathrm{CAC}$ and branches in the yak displayed no significant differences from that in cattle and calibers of the CCA also showed no significant discrepancy between the two species. Due to some studies, however the average brain weight $(337 \mathrm{~g})$ of the yak (Zhang, 1989) was only $67.4 \%$ of that of cattle $(500 \mathrm{~g})$ (Sisson, 1914) as far as per unit brain weight is concerned which indicated the CCA and IAV in the yak were more developed, so researchers estimated that blood pressure of the CCA will become lower and the IAV might be able to receive more blood from the ERM. Therefore, we proposed that the blood volume per unit brain tissue in the yak was more abundant than those in cattle and the conclusion could be confirmed by the reports on the effects of prolonged hypoxia in rat brain (Lauro and Lamanna, 1997) so that additional blood flow will be received by more capillary. These peculiarities mentioned above accounted for the small brain volume in the yak had more developed IAV which was more beneficial to supplying blood to the brain and perhaps be one of the adaptive mechanisms of the yak on plateau environment.

Function of the yak's ERM: The ERM was a striking arterial system that was observed in most artiodactyls, including cattle, sheep, goats and pigs (Ocal and Aslan, 1994; Khamas and Ghoshal, 1985; Edelman et al., 1972; Ghoshal and Khamas, 1985). Present results showed that the yak and cattle had developed ERM. By comparison, however researchers found that the area of the anterior ERM was larger and more extensive, the posterior joint section and the posterior ERM were also more developed in the yak, even the anastomotic branches of the ERM were more abundant and their gross cross area were also larger than those in cattle. So, we considered that more extensive anterior ERM and developed posterior joint section in the yak were beneficial to exchanging and complementing blood from lobe to lobe and the developed posterior ERM was beneficial to preservation and transportation of blood. Moreover, more anastomotic branches mean supply more blood to the ERM.

As to function of the ERM, different researchers stated different opinions. In some studies, this unique arterial system was thought to regulate blood temperature principally as the blood passes through the arterial meshwork of the rete in close association with the cavernous sinus, thereby protecting the brain (Jessen, 2001). It was reported that two-humped camel had developed ERM-a heat exchange device which had evolved for adapting extreme environment (Wang, 1989). Many researchers also agreed that ERM was able to mechanically change the hemodynamics of the circulation (Uehara et al., 1978).

In present study, researchers proposed that the ERM should be a vital organ of regulating and improving blood supply and regarded as a device of buffering and stabilizing blood pressure in the course of supplying blood to the brain. Nevertheless because the most regions of the ERM and cavernous sinus were overlapping, its brain-cooling function was not able to be ignored. 
Anatomical superiority of the CAS: Changes of vascular caliber become the main factor determining blood flow if the differential pressure of artery and vein would be similar. Some studies pointed out that the ratio of the blood supply to ERM can be evaluated by the diameter of each vessel cast of the afferent arteries. A study about the blood flow distribution of the ERM in one-humped camel showed that the branches of the maxillary artery carried $76.41 \%$ of gross blood supply in the ERM (Ocal et al., 1998). Researchers found that diameters of main arteries of the EAV showed no significant differences between the yak and cattle but the caliber's decreasing gradient of some arteries in the yak were milder than that in cattle which accounted for more blood flow can enter into the ERM via the EAV.

Although, the blood volume of the IAV in the yak seemed less, the blood volume of the developed ERM was almost $125.6 \%$ of those of cattle. Therefore the gross blood volume of ERM and IAV reached $96.6 \%$ of those of cattle. The results illustrated that the CAS especially ERM in the yak showed anatomical superiority and can preserve and supply more blood for brain tissue. In this sense, regardless of significantly different body and brain weights, we deduced that relative gross blood volume of the IAV and ERM in the yak was larger than that in cattle, so sufficient blood supply to the brain tissue of the yak can be guaranteed. This might be another one of adaptive mechanisms of the CAS in the yak on the harsh areas.

\section{CONCLUSION}

Although, the general features and most of arterial calibers of the CAS in two species were similar, in comparison to low-altitude cattle, the anterior-posterior diameter of the CAC was shorter and interval distance of the CCA was closer in the plateau yak. In addition, results showed that the yak has more developed IAV and ERM than that in cattle and the blood volume of the CAS in the yak showed anatomical superiority. Researchers considered that the anatomic peculiarities of the CAC and relatively developed IAV in the yak were beneficial to supplying more blood to the brain. The yak's developed ERM was advantageous of preservation, exchange and complement of the blood and it might play a vital role in buffering and regulating cerebral blood flow so as to meet demand of the yak's brain for blood supply in the plateau areas.

\section{ACKNOWLEDGEMENTS}

Researchers thank Xin Cai from the Southwest University of Science and Technology, Binyun Ma from the Lanzhou University and Hui Zhu, Ganggang Zhang from the Gansu Agricultural University for all their support. This research project was fully sponsored by the National Natural Science Foundation with grant number 30960164.

\section{REFERENCES}

Baldwin, B.A. and F.R. Bell, 1963. The anatomy of the cerebral circulation of the sheep and ox. The dynamic distribution of the blood supplied by the carotid and vertebral arteries to cranial regions. J. Anat, 97: 203-215.

Baldwin, B.A., 1964. The anatomy of the arterial supply to the cranial regions of the sheep and ox. Am. J. Anat, 115: 101-117.

Cui, Y., Y.Y. He and J. Wang, 2008. Morphological characteristics of the ventricular microvasculature in adult yak. Acta Theriologica Sinica., 28: 300-304.

Du, X.X., 2008. Study on the construction characteristics of pulmonary microvasculature in yak of different ages and adult cattle. Ph.D. Thesis, Gansu Agricultural University. Lanzhou. China.

Edelman, N.H., P. Epstein, N.S. Cherniak and A.P. Fishman, 1972. Control of cerebral blood flow in the goat; role of the carotid rete. Am. J. Physiol., 223: 615-619.

Ghoshal, N.G. and W. Khamas, 1985. Gross and histomorphological study on the rostral epidural rete mirabile of the pig. Indian J. Anim. Sci., 55: 304-310.

He, Y.Y., 2007. Studies on the microvasculature of in infant and adult yak's heart. M.D. Thesis, Gansu Agricultural University, Lanzhou, China.

Huang, S.Y., S. Sun, T. Droma, J. Zhuang and J.X. Tao et al., 1992. Internal carotid arterial flow velocity during exercise in Tibetan and Han residents of Lhasa (3,658m). J. Applied Physiol., 73: 2638-2642.

Jansen, G.F. and B. Basnyat, 2011. Brain blood flow in Andean and Himalayan high-altitude populations: Evidence of different traits for the same environmental constraint. J. Cereb. Blood Flow Metab., 31: 706-714.

Jessen, C., 2001. Selective brain cooling in mammals and birds. Jpn. J. Physiol., 51: 291-301.

Khamas, W.A. and N.G. Ghoshal, 1985. Gross and scanning electron microscopy of the carotid retecavernous sinus complex of the sheep (Ovis aries). Anat. Anz., 159: 173-179.

Kurtul, I., K. Aslan, S. Ozcan and G. Aksoy, 2003. Formation of cerebral arterial circle (Circulus arteriosus cerebri) in the fetus of zavot-bred cattle. Kafkas Univ. Vet. Fak. Derg., 9: 153-156. 
Lalthantluanga, R., H. Wiesner and G. Braunitzer, 1985. Studies on yak hemoglobin (Bos grunniens, Bovidae): Structural basis for high intrinsic oxygen affinity? Biol. Chem., 366: 63-68.

Lauro, K.L. and J.C. Lamanna, 1997. Adequacy of cerebral vascular remodeling following three weeks of hypobaric hypoxia. Examined by an integrated composite analytical model. Adv. Exp. Med. Biol., 411: 369-376.

Ocal, M.K. and K. Aslan, 1994. A putative study on the retinal arteries in the bovine fetus. Ann. Anat, 176: 151-153.

Ocal, M.K., H. Erden, I. Ogut and M.E. Kara, 1998. A quantitative study on the retial arteries in onehumped camels. Ann. Anat., 180: 369-371.

Sisson, S., 1914. The Anatomy of the Domestic Animals. 2nd Edn., W.B. Saunders Company, Philadelphia, London, pp: 980-981.

Shwini, C., R. Shubha and K. Jayanthi, 2008. Comparative anatomy of the circle of Willis in man, cow, sheep, goat and pig. Neuroanatomy, 7: 54-65.

Steven, D.H., 1964. The distribution of external and internal ophthalmic arteries in the ox. J. Anat, 98: 429-435.
Tanaka, H., N. Fujita, T. Enoki, K. Matsumoto, Y. Watanabe, K. Murase and H. Nakamura, 2006. Relationship between variations in the circle of Willis and flow rates in internal carotid and basilar arteries determined by means of magnetic resonance imaging with semiautomated lumen segmentation: Reference data from 125 healthy volunteers. AJNR Am. J. Neuroradiol., 27: $1770-1775$.

Uehara, M., N. Kudo and M. Sugimura, 1978. Morphological studies on the rete mirabile epidural in the calf. Jpn. J. Vet. Res., 26: 11-18.

Wang, W.H., 1989. The carotid rete of the camel (Bactrianus camelus). Chinese J. Anim. Vet. Sci., 20: $90-95$.

Wang, X.R., Y. Liu, L.P. Zhang, X.J. Wang and J.P. Wu, 2012. Comparative anatomical study of the epidural retia mirabile in the Yak and cattle. Asian J. Anim. Vet. Adv., 7: 884-890.

Zhang, R.C., 1989. Yak in China. Gansu Science and Technology Press (China), Lanzhou., pp: 96-102. 